\title{
Efecto del acceso al tratamiento de la periodontitis sobre la calidad de vida relacionada con la salud bucal
}

\section{Effect of the access to periodontitis treatment on oral health related quality of life}

\author{
Renata De La Hoz-Perafán (D)1, Erika Loaiza-Echeverri (iD)2, Ivan Arroyave (D)3 \\ 1. Universidad del Magdalena. Santa Marta, Colombia. Universidad CES. Medellín, Colombia. Correo: rdelahoz@unimagdalena.edu.co - \\ https://orcid.org/0000-0002-4411-3029 \\ 2. Universidad CES. Medellín, Colombia. Correo: etloaiza@ces.edu.co - https://orcid.org/0000-0003-3589-6786 \\ 3. Universidad de Antioquia. Medellín, Colombia. Correo: ivan.arroyave@udea.edu.co - https://orcid.org/0000-0001-9989-5833
}

Tipología: Artículo de investigación científica y tecnológica

Para citar este artículo: De la Hoz RP, Loaiza E, Arroyave I. Efecto del acceso al tratamiento de la periodontitis sobre la calidad de vida relacionada con la salud bucal. 2021 septiembre; 18(3 número especial): 53-68. Doi: https://doi.org/10.21676/2389783X.4263

Recibido en abril 19 de 2021

Aceptado en junio 29 de 2021

Publicado en línea en agosto 24 de 2021

\section{Palabras clave: odontología; enfermedad periodontal; acceso salud; salud pública; epidemiología.}

\section{RESUMEN}

La calidad de vida relacionada con la salud oral puede ser influenciada por la periodontitis, una enfermedad inflamatoria que provoca pérdida dental y afecta la integridad y funcionalidad de la cavidad bucal; su condición más severa se presenta en $11,2 \%$ de la población mundial. En Colombia, su tratamiento no está cubierto por el Plan de Beneficios en Salud, la presencia de barreras de acceso se asocia con estrés y depresión afectando negativamente la calidad de vida. El objetivo de este estudio fue determinar el efecto del acceso al tratamiento de la periodontitis sobre la calidad de vida relacionada con la salud bucal. Se utilizó el diseño de seguimiento a una cohorte, por censo se reclutaron 149 participantes con periodontitis. Se dividieron entre quienes lograron o no acceder al tratamiento, se midió calidad de vida al inicio y al final del seguimiento. Se encontró asociación entre la calidad de vida relacionada con salud bucal con las variables sexo y uso de prótesis dental. La ausencia del tratamiento especializado de la enfermedad no afecta la calidad de vida porque los usuarios del centro de salud no perciben las barreras de acceso administrativas y organizacionales.

\section{ABSTRACT}

\section{Keywords:} Dentistry; Periodontal disease; Health care access; Public health; Epidemiology.
The oral health related quality of life can be influenced by periodontitis, an inflammatory disease that causes tooth loss and affects the oral integrity and functionality. Its most severe condition, it occurs in $11.2 \%$ of the world population. In Colombia, the periodontitis treatment is not covered by the public insurance. The presence of barriers to the periodontal treatment access could be associated with stress and depression, negatively affecting the quality of life. The objective of this study was to determine the effect of the periodontitis treatment access on the oral health related quality of life. A cohort follow-up design was used, 149 participants were recruited by census. They were divided between those who were able or not to access the treatment, quality of life was measured at the beginning and at the end of the follow-up. Association was found between the oral health related quality of life and the variables sex and use of dental prostheses. The absence of periodontal specialized treatment does not affect the quality of life because the health care center users do not perceive the administrative and organizational access barriers. 


\section{INTRODUCCIÓN}

La calidad de vida relacionada con la salud bucal (CVRSB) es un complejo mutidimensional que incluye un componente físico y otro psicológico ${ }^{1}$. Es afectada por diferentes factores como sexo, nivel educativo, ingresos financieros y la edad, por condicionar el papel del individuo en la sociedad. Dentro de las enfermedades crónicas que han sido asociadas a la CVRSB por parte de los pacientes se encuentra la periodontitis, que lleva a la pérdida dental; esta enfermedad es la condición de carácter crónico e inflamatorio más común en el mundo, su prevalencia en su condición más grave afecta el $11,2 \%$ de la población ${ }^{2,3}$. En Colombia, la periodontitis presenta un comportamiento similar al reportado a nivel mundial, el último estudio en salud bucal evidenció como la mayor parte de la población $(61,8 \%)$ fue diagnosticada con periodontitis en sus diferentes grados de severidad, siendo la más frecuente la moderada presente en el $43,46 \%$ de la población, seguida por un $10,62 \%$ con periodontitis grave, encontrada con mayor frecuencia en hombres, y finalmente el 7,72\% presentó leve ${ }^{4}$.

Las secuelas de la periodontitis pueden llegar a influir en el paciente afectando su desempeño social relacionado a la sonrisa, por favorecer la creación estrategias para ocultar los dientes y la sonrisa, además del temor constante a la exposición por la halitosis o a ser estigmatizados por la apariencia de sus encías cuando la enfermedad está activa, esto puede desencadenar dificultades en las relaciones laborales e íntimas como causa del sentimiento de vergüenza, que deriva en desventajas durante el desarrollo de la vida cotidiana, incluso se mencionan impactos importantes como la incapacidad para participar en pasatiempos, compartir tiempo con la familia, socializar y disfrutar de los días de fiestas ${ }^{2}$. Gift et $a l^{1}$, ponen en consideración, la naciente preocupación, estrés y/o depresión que se puede generar cuando un paciente no logra acceder al tratamiento, lo que permite considerar la atención de la enfermedad como un factor que logra influir sobre la percepción de la calidad de vida.

En Colombia el tratamiento de la periodontitis no está cubierto por el Plan de beneficios en Salud
(PBS), los pacientes en la búsqueda del tratamiento de la enfermedad en ocasiones deben recurrir a estrategias legales a menos que tenga los recursos financieros para acceder de manera particular. La ausencia de investigaciones sobre el tema no permite soportar con evidencia las observaciones sobre estos hallazgos, debido a que se da relevancia al acceso en los servicios de salud. En este estudio se define acceso al tratamiento como el ingreso del individuo al sistema de salud por la necesidad de atención, se incluyeron los factores que inhiben o favorecen la entrada inicial al sistema para recibir la atención requerida, y tener en cuenta la satisfacción del paciente para decidir si continua la utilización de los servicios ${ }^{5}$. Las necesidades de atención en salud insatisfechas serán el indicador proxy representativo del acceso $0^{6,7}$, un paciente que accede al tratamiento de la periodontitis será un paciente satisfecho con la atención recibida en la IPS. Los colombianos no tienen en su Plan de Beneficios en Salud (PBS) acceso a los tratamientos periodontales especializados como alternativa terapéutica, ubicándolos en una situación de riesgo para su bienestar $^{8,9}$; al considerar la prevalencia de la enfermedad y las variadas experiencias en su manejo clínico, el objetivo de este estudio es determinar el efecto del acceso al tratamiento de la periodontitis sobre la calidad de vida relacionada con la salud bucal.

\section{MATERIALES Y MÉTODOS}

\section{Tipo de Investigación}

El enfoque de la investigación es cuantitativo, el diseño de observacional de seguimiento a una cohorte. En una primera fase se evaluaron las propiedades psicométricas de replicabilidad de la versión chilena ${ }^{10}$ del Oral Health Impact Profile -49 (OHIP-49) con 207 participantes $^{11}$, el cual, luego de la prueba piloto fue reemplazado por OHIP-14 con 145 participantes, ninguno de estos instrumentos había sido validado en Colombia hasta el inicio del estudio ${ }^{12}$. En esta fase, los participantes fueron reclutados en una clínica privada de la ciudad y en una IPS universitaria, con la finalidad de ampliar la variabilidad de los factores socioeconómicos incluidos, la recolección de datos se realizó entre 
2017 y 2018, para ambos el tamaño de muestra fue el sugerido por la Comisión Internacional del Test ${ }^{13}$.

\section{Participantes}

Durante los años 2019 y 2020 se ejecutó la segunda fase, se reclutaron por Censo 149 participantes mayores de edad en una IPS perteneciente al régimen subsidiado en la ciudad de Santa Marta, Colombia. Se incluyeron pacientes con al menos 18 dientes en boca y diagnóstico de periodontitis según los criterios de $\mathrm{Eke}^{14}$; se excluyeron gestantes, pacientes con tratamiento activo, y con discapacidades cognitivas. En el equipo de trabajo de campo se incluyó un odontólogo para diagnosticar enfermedad periodontal $y$ estandarizado con un especialista en periodoncia (índice Kappa=0,82); los otros miembros del equipo fueron un encuestador y el investigador principal, todos recibieron entrenamiento por el área de psicología.

\section{Instrumento}

Se utilizó el OHIP-14 para conocer la percepción de calidad de vida relacionada con salud bucal en pacientes con periodontitis. En esta investigación, la mayor afectación negativa (mayor puntaje) se presentará en términos de "peor calidad de vida relacionada con la salud bucal", y en el caso de la menor afectación negativa (menor puntaje) será descrita como una "mejor calidad de vida relacionada con la salud bucal". Slade ${ }^{15,16}$, implementó para el OHIP-49 y OHIP-14 el uso del umbral "a veces", "frecuentemente" y "siempre" como peor calidad de vida, mientras que "nunca" $y$ "a veces" representan mejor CVRSB, para indicar que los participantes del estudio habían experimentado al menos sutilmente el evento de interés.

\section{Procedimiento}

Se realizó una medición inicial de CVRSB cuando se diagnosticó la enfermedad, una segunda medición telefónica posterior al proceso de acceso al tratamiento de la periodontitis, en un tercer momento de medición presencial, se hizo la segunda medición de CVRSB, para un total de tres mediciones en la cohorte. Posteriormente se dividieron en dos grupos, quienes lograron acceder y los que no lo hicieron, en cada uno se midió la CVRSB. Se midieron en total 61 variables, se utilizó análisis estadístico descriptivo, las asociaciones se establecieron con la prueba estadística $\mathrm{Chi}^{2}$ de Pearson, la prueba t-student y en el caso de no tener distribución normal se aplicó la prueba $U$ de MannWhitney; las diferencias entre la medición inicial y final de la CVRSB se analizó con el test de McNemar y el análisis multivariado con regresión Log binomial de los modelos lineales generalizados para soportar el alcance explicativo de acuerdo al diseño longitudinal del estudio. Para el análisis estadístico se utilizaron los softwares estadísticos SPSS v26 y Jamovi.

\section{Declaración sobre aspectos éticos}

Este estudio adaptó las Pautas éticas internacionales para la investigación biomédica en seres humanos bajo los principios de respeto por las personas, beneficencia y justicia; la resolución 008430 de 1993 del Ministerio de Salud, Resolución 1995 de 1999 sobre manejo de historias clínicas, y la Ley Estatutaria 1581 del 2012 para protección de datos personales. La investigación fue avalada por el Comité de Ética para Investigación en Humanos de la Universidad CES (código 510) en la sesión 87 del 2015 y renovada en la sesión 123 del 2018; el Comité aprobó el consentimiento informado y acuerdos de asentimiento utilizados en el estudio.

\section{RESULTADOS}

Se encontró mejor comportamiento de las propiedades psicométricas del OHIP-14 en lo concerniente a alfa de Cronbach $(0,83)$, porcentajes de éxito tanto de consistencia interna como de poder discriminante, esto, al compararlos con los resultados del OHIP-49 (alfa Cronbach 0,95), por eso se seleccionó el OHIP-14 para la segunda fase. Durante el periodo de reclutamiento se realizaron un total de 436 sondajes periodontales, de los cuales 229 fueron diagnosticados con periodontitis y reclutados para participar en el estudio, lo que equivale al $52,52 \%$ del total de examinados; al finalizar el tiempo de seguimiento de la cohorte, de 229 participantes un total de 149 completaron la encuesta en sus tres momentos de medición lo que 
equivale a una pérdida en el seguimiento del 34,93\% de los participantes.

De los 149 participantes que finalizaron el seguimiento, al hacer la revisión de las historias clínicas, se encontró que solo cuatro tenían diagnóstico previo de periodontitis antes de ingresar al estudio, lo que equivale a un $2,7 \%$ de la muestra, de estos, 101 (67,8\%) tuvieron otros tipos de diagnósticos como caries, gingivitis crónica, y gingivitis aguda que fue la más frecuente; por otro lado, 29 (19,5\%) de ellos no tenían ningún tipo de diagnóstico en su historia clínica, los 15 (10,1\%) restantes estaban sin registro de atención odontológica. Lo anterior, evidencia el subregistro del $97,31 \%$ de los casos de periodontitis atendidos en el servicio de odontología de la IPS.

El $65,8 \%$ tenía edad entre 18 y 44 años; $32,9 \%$ edades entre 45 y 65 años; participaron 35 hombre y 114 mujeres; la mayoría cursaron primaria y secundaria; la mayoría pertenecen al estrato 1 socioeconómico, seguido por el estrato 2 ; en esta población, los niveles de escolaridad más frecuentes fueron primaria y bachillerato. La mayoría de los participantes se tenían como ocupación el hogar, o eran trabajadores independientes, además del grupo de desempleados. La periodontitis en estadio moderado fue la condición más frecuente, seguido por periodontitis leve y grave (Tabla 1). Esta población tuvo baja frecuencia de asistencia al odontólogo (Tabla 2).

En la tabla 3 se observa que los participantes refieren preocuparse por sus dientes y encías, se sienten satisfechos con su apariencia y con sí mismos. En lo referente al servicio, tienen accesibilidad al centro de salud, la obtención de citas y en general están satisfechos con las distintas áreas del servicio que permiten recibir manejo clínico ante la necesidad de atención que manifiestan (Tabla 4).

Las dimensiones que presentaron con mayor frecuencia peor CVRSB fueron dolor físico y malestar psicológico como se observa en la tabla 5.

Los resultados de la prueba de McNemar, evidenciaron que en la mayoría de las dimensiones no se encontraron diferencias estadísticamente significativas entre la medición inicial y final de la CVRSB; pero los ítems y dimensiones que tuvieron cambio significativo entre la primera y segunda medición de la calidad de vida fueron la dimensión Incapacidad Física y sus dos ítems que son: tener una dieta no satisfactoria $(p=<0,001)$, y el ítem tener que interrumpir comidas por su condición bucal, ambos ítems de esta dimensión mostraron cambios entre las dos mediciones $(p=0,005)$. Otra dimensión que presentó cambios en sus mediciones inicial y final de CVRSB fue incapacidad psicológica, pero solo en su ítem encontrar difícil relajarse por su condición bucal $(p=0,010)$.

Al calcular la puntuación total de la segunda medición del OHIP-14 a través del método aditivo simple ${ }^{17,18}$; con base en el estadístico de 5,14 $(p=0,07)$ obtenido con la prueba Kruskal-Wallis (ver tabla 6), se considera que la calidad de vida relacionada con la salud bucal en los distintos grupos de severidad de la periodontitis, presentan datos con la misma distribución. Se puede considerar una mejor calidad de vida los valores $\leq 12$, una afectación media de la CVRSB con valores $\leq 14$ o iguales a 13, y para representar peor CVRSB los valores $\geq 15$. Para el análisis multivariado, se determinó que de forma global los valores $\leq 12$ representan una mejor CVRSB, mientras que los valores $\geq 13$ representan una peor CVRSB. De esta manera se estableció que 85 (57\%) participantes presentaron una mejor CVRSB, mientras que 64 participantes (43\%) tuvieron una peor CVRSB.

Un total de nueve variables presentaron asociación estadísticamente significativa $(p<0,05)$ con peor CVRSB, estas variables ingresan a la regresión Log Binomial de los modelos lineales generalizados. Con base en la literatura se decidió incluir las variables recibir tratamiento de la enfermedad, la variable ocupación por su representación del poder adquisitivo de los participantes y su nivel de vida en esta población donde prevalece el estrato socioeconómico uno, y la variable proxy de acceso. Las variables incluidas en el estudio y referentes al acceso al tratamiento de la periodontitis no fueron estadísticamente significativas en el análisis bivariado o multivariado. Finalmente se incluyeron un total de 12 variables en el análisis multivariado como se observa en la tabla 7. 
Tabla 1. Distribución porcentual de las variables demográficas, socioeconómicas.

\begin{tabular}{|c|c|c|c|c|c|c|c|c|c|}
\hline & & \multicolumn{2}{|c|}{ Total } & \multicolumn{2}{|c|}{$\begin{array}{l}\text { Periodontitis } \\
\text { leve }\end{array}$} & \multicolumn{2}{|c|}{$\begin{array}{l}\text { Periodontitis } \\
\text { moderada }\end{array}$} & \multicolumn{2}{|c|}{$\begin{array}{l}\text { Periodontitis } \\
\text { grave }\end{array}$} \\
\hline & & $\mathrm{n}$ & $\%$ & $n$ & $\%$ & $\mathrm{n}$ & $\%$ & $\mathrm{n}$ & $\%$ \\
\hline Población & & 149 & $(100)$ & 44 & $(29,5)$ & 72 & $(48,3)$ & 33 & $(22 \%)$ \\
\hline \multirow{3}{*}{ Edad } & 18-44 años & 98 & $(65,8)$ & 39 & $(26,2)$ & 47 & $(31,5)$ & 12 & $(8,1)$ \\
\hline & 45-65 años & 49 & $(32,9)$ & 5 & $(3,4)$ & 24 & $(16,1)$ & 20 & $(13,4)$ \\
\hline & $>65$ años & 2 & $(1,3)$ & 0 & (0) & 1 & $(0,7)$ & 1 & $(0,7)$ \\
\hline \multirow[t]{2}{*}{ Sexo } & Masculino & 35 & $(23,5)$ & 8 & $(5,4)$ & 16 & $(10,7)$ & 11 & $(7,4)$ \\
\hline & Femenino & 114 & $(76,5)$ & 36 & $(24,2)$ & 56 & $(37,6)$ & 22 & $(14,8)$ \\
\hline \multirow{5}{*}{ Estado civil } & Soltero & 43 & $(28,9)$ & 15 & $(10,1)$ & 20 & $(13,4)$ & 8 & $(5,4)$ \\
\hline & Casado & 29 & $(19,5)$ & 8 & $(5,4)$ & 12 & $(8,1)$ & 9 & $(6,0)$ \\
\hline & Viudo & 3 & $(2,0)$ & 0 & (0) & 1 & $(0,7)$ & 2 & $(1,3)$ \\
\hline & Separado & 2 & $(1,3)$ & 2 & $(1,3)$ & 0 & (0) & 0 & (0) \\
\hline & Unión libre & 72 & $(48,3)$ & 19 & $(12,8)$ & 39 & $(26,2)$ & 14 & $(9,4)$ \\
\hline \multirow{5}{*}{ Nivel de escolaridad } & Sin educación & 6 & $(4,0)$ & 2 & $(1,3)$ & 2 & $(1,3)$ & 2 & $(1,3)$ \\
\hline & Primaria & 55 & $(36,9)$ & 12 & $(8,1)$ & 27 & $(18,1)$ & 16 & $(10,7)$ \\
\hline & Secundaria & 52 & $(34,9)$ & 15 & $(10,1)$ & 24 & $(16,1)$ & 13 & $(8,7)$ \\
\hline & Técnico & 31 & $(20,8)$ & 12 & $(8,1)$ & 17 & $(11,4)$ & 2 & $(1,3)$ \\
\hline & Universitario & 5 & $(3,4)$ & 3 & $(2,0)$ & 2 & $(1,3)$ & 0 & $(0)$ \\
\hline \multirow{5}{*}{$\begin{array}{l}\text { Estrato } \\
\text { socioeconómico }\end{array}$} & 0 & 2 & $(1,3)$ & 0 & (0) & 1 & $(0,7)$ & 1 & $(0,7)$ \\
\hline & 1 & 106 & $(71,1)$ & 35 & $(23,5)$ & 52 & $(34,9)$ & 19 & $(12,8)$ \\
\hline & 2 & 27 & $(18,1)$ & 5 & $(3,4)$ & 14 & $(9,4)$ & 8 & $(5,4)$ \\
\hline & 3 & 12 & $(8,1)$ & 3 & $(2,0)$ & 4 & $(2,7)$ & 5 & $(3,4)$ \\
\hline & 4 & 2 & $(1,3)$ & 1 & $(0,7)$ & 1 & $(0,7)$ & 0 & $(0)$ \\
\hline \multirow{5}{*}{ Ocupación } & Desempleado & 31 & $(208)$ & 10 & $(67)$ & 15 & $(101)$ & 6 & $(40)$ \\
\hline & Empleado & 15 & $(10,1)$ & 5 & $(3,4)$ & 9 & $(6,0)$ & 1 & $(0,7)$ \\
\hline & Hogar & 61 & $(40,9)$ & 18 & $(12,1)$ & 31 & $(20,8)$ & 12 & $(8,1)$ \\
\hline & Independiente & 34 & $(22,8)$ & 6 & $(4,0)$ & 15 & $(10,1)$ & 13 & $(8,7)$ \\
\hline & Estudiante & 8 & $(5,4)$ & 5 & $(3,4)$ & 2 & $(1,3)$ & 1 & $(0,7)$ \\
\hline \multirow{6}{*}{ Compromiso sistémico } & No refiere & 116 & $(77,9)$ & 37 & $(24,8)$ & 58 & $(38,9)$ & 21 & $(14,1)$ \\
\hline & Diabetes & 4 & $(2,7)$ & 1 & $(0,7)$ & 1 & $(0,7)$ & 2 & $(1,3)$ \\
\hline & Hipertensión & 18 & $(12,1)$ & 3 & $(2,0)$ & 8 & $(5,4)$ & 7 & $(4,7)$ \\
\hline & $\begin{array}{l}\text { Enfermedad } \\
\text { renal }\end{array}$ & 1 & $(0,7)$ & 1 & $(0,7)$ & 1 & $(0,7)$ & 1 & $(0,7)$ \\
\hline & $\mathrm{VIH}$ & 2 & $(1,3)$ & 0 & (0) & 1 & $(0,7)$ & 1 & $(0,7)$ \\
\hline & Otros & 8 & $(5,4)$ & 4 & $(2,7)$ & 2 & $(1,3)$ & 1 & $(0,7)$ \\
\hline
\end{tabular}


Tabla 2. Distribución porcentual de las variables clínicas.

\begin{tabular}{|c|c|c|c|c|c|c|c|c|c|}
\hline & & \multicolumn{2}{|c|}{ Total } & \multicolumn{2}{|c|}{$\begin{array}{l}\text { Periodontitis } \\
\text { leve }\end{array}$} & \multicolumn{2}{|c|}{$\begin{array}{l}\text { Periodontitis } \\
\text { moderada }\end{array}$} & \multicolumn{2}{|c|}{$\begin{array}{l}\text { Periodontitis } \\
\text { grave }\end{array}$} \\
\hline & & $\mathrm{n}$ & $\%$ & $\mathrm{n}$ & $\%$ & $n$ & $\%$ & $\mathrm{n}$ & $\%$ \\
\hline \multirow[t]{2}{*}{ Uso de prótesis dental } & $\mathrm{Si}$ & 9 & $(6,0)$ & 2 & $(1,3)$ & 3 & $(2,0)$ & 4 & $(2,7)$ \\
\hline & No & 140 & $(94,0)$ & 42 & $(28,2)$ & 69 & $(46,3)$ & 29 & $(19,5)$ \\
\hline \multirow{7}{*}{$\begin{array}{l}\text { Última visita al } \\
\text { odontólogo }\end{array}$} & Una semana & 12 & (81) & 6 & $(40)$ & 3 & $120)$ & 3 & $120)$ \\
\hline & Un mes & 19 & $(12,8)$ & 5 & $(3,4)$ & 12 & $(8,1)$ & 2 & $(1,3)$ \\
\hline & Tres meses & 17 & $(11,4)$ & 8 & $(5,4)$ & 5 & $(3,4)$ & 4 & $(2,7)$ \\
\hline & Seis meses & 16 & $(10,7)$ & 3 & $(2,0)$ & 9 & $(6,0)$ & 4 & $(2,7)$ \\
\hline & Un año & 27 & $(18,1)$ & 7 & $(4,7)$ & 13 & $(8,7)$ & 7 & $(4,7)$ \\
\hline & Más de un año & 56 & $(37,6)$ & 15 & $(10,1)$ & 29 & $(19,5)$ & 12 & $(8,1)$ \\
\hline & $\begin{array}{l}\text { Asiste por } \\
\text { primera vez }\end{array}$ & 2 & $(1,3)$ & 0 & (0) & 1 & $(0,7)$ & 1 & $(1,7)$ \\
\hline \multirow{4}{*}{$\begin{array}{l}\text { Actividad realizada en } \\
\text { su última visita al } \\
\text { odontólogo }\end{array}$} & $\begin{array}{l}\text { Consulta } \\
\text { diagnóstica }\end{array}$ & 7 & $(4,7)$ & 1 & $(0,7)$ & 5 & $(3,4)$ & 1 & $(0,7)$ \\
\hline & Higiene oral & 114 & $(76,5)$ & 35 & $(23,5)$ & 52 & $(34,9)$ & 27 & $(18,1)$ \\
\hline & $\begin{array}{l}\text { Tratamiento } \\
\text { caries }\end{array}$ & 20 & $(13,4)$ & 7 & $(4,7)$ & 9 & $(6,0)$ & 4 & $(2,7)$ \\
\hline & Exodoncia & 6 & $(4,0)$ & 1 & $(0,7)$ & 5 & $(3,4)$ & 0 & (0) \\
\hline \multirow[t]{2}{*}{ Diagnóstico de caries } & $\mathrm{Si}$ & 49 & $(32,9)$ & 21 & $(14,1)$ & 20 & $(13,4)$ & 8 & $(5,4)$ \\
\hline & No & 100 & $(67,1)$ & 23 & $(15,4)$ & 52 & $(34,9)$ & 25 & $(16,8)$ \\
\hline \multirow[t]{2}{*}{ Pérdidas dentales } & $\mathrm{Si}$ & 110 & $(73,8)$ & 28 & $(18,8)$ & 51 & $(34,2)$ & 31 & $(20,8)$ \\
\hline & No & 39 & $(26,2)$ & 16 & $(10,7)$ & 21 & $(14,1)$ & 2 & $(1,3)$ \\
\hline \multirow{3}{*}{$\begin{array}{l}\text { Movilidad dental, } \\
\text { lesión de furca, o } \\
\text { supuración }\end{array}$} & & & & & & & & & \\
\hline & $\mathrm{Si}$ & 16 & $(10,7)$ & 1 & $(0,7)$ & 3 & $(2,0)$ & 12 & $(8,1)$ \\
\hline & No & 133 & $(89,3)$ & 43 & $(28,9)$ & 69 & $(46,3)$ & 21 & $(14,1)$ \\
\hline
\end{tabular}


Tabla 3. Distribución porcentual de las variables de acceso al servicio de odontología según el nivel de severidad de la periodontitis.

\begin{tabular}{|c|c|c|c|c|c|c|c|c|c|}
\hline & & \multicolumn{2}{|c|}{ Total } & \multicolumn{2}{|c|}{$\begin{array}{l}\text { Periodontitis } \\
\text { leve }\end{array}$} & \multicolumn{2}{|c|}{$\begin{array}{l}\text { Periodontitis } \\
\text { moderada }\end{array}$} & \multicolumn{2}{|c|}{$\begin{array}{l}\text { Periodontitis } \\
\text { grave }\end{array}$} \\
\hline & & $\mathrm{n}$ & $\%$ & $\mathrm{n}$ & $\%$ & $\mathrm{n}$ & $\%$ & $\mathrm{n}$ & $\%$ \\
\hline Población & & 149 & $(100)$ & 44 & $(29,5)$ & 72 & $(48,3)$ & 33 & (22\%) \\
\hline \multirow{3}{*}{$\begin{array}{l}\text { ¿Se ha sentido tan mal con } \\
\text { su condición oral que ha } \\
\text { llegado a llorar? }\end{array}$} & & & & & & & & & \\
\hline & $\mathrm{Si}$ & 76 & $(51,0)$ & 27 & $(18,1)$ & 31 & $(20,8)$ & 18 & $(12,1)$ \\
\hline & No & 73 & $(49,0)$ & 17 & $(11,4)$ & 41 & $(27,5)$ & 15 & $(10,1)$ \\
\hline \multicolumn{10}{|l|}{ ¿Se ha sentido alguna vez } \\
\hline \multirow{2}{*}{$\begin{array}{l}\text { acomplejado por sus } \\
\text { dientes y/o encías? }\end{array}$} & $\mathrm{Si}$ & 41 & $(27,5)$ & 13 & $(8,7)$ & 12 & $(8,1)$ & 16 & $(10,7)$ \\
\hline & No & 108 & $(72,5)$ & 31 & $(20,8)$ & 60 & $(40,3)$ & 17 & $(11,4)$ \\
\hline \multirow{3}{*}{$\begin{array}{l}\text { ¿Considera que sus dientes } \\
\text { y/o encías tienen buena } \\
\text { apariencia? }\end{array}$} & & & & & & & & & \\
\hline & $\mathrm{Si}$ & 93 & $(62,4)$ & 25 & $(16,8)$ & 50 & $(33,6)$ & 18 & $(12,1)$ \\
\hline & No & 56 & $(37,6)$ & 19 & $(12,8)$ & 22 & $(14,8)$ & 15 & $(10,1)$ \\
\hline \multirow{3}{*}{$\begin{array}{l}\mathrm{¿Se} \text { siente satisfecho } \\
\text { consigo mismo en general? }\end{array}$} & & & & & & & & & \\
\hline & $\mathrm{Si}$ & 141 & $(94,6)$ & 41 & $(27,5)$ & 69 & $(46,3)$ & 31 & $(20,8)$ \\
\hline & No & 8 & $(5,4)$ & 3 & $(2,0)$ & 3 & $(2,0)$ & 2 & $(1,3)$ \\
\hline \multirow{3}{*}{$\begin{array}{lcc}\text { ¿Considera que su } \\
\text { apariencia personal le } \\
\text { facilita alcanzar sus metas? }\end{array}$} & & & & & & & & & \\
\hline & $\mathrm{Si}$ & 112 & $(75,2)$ & 32 & $(21,5)$ & 54 & $(36,2)$ & 26 & $(17,4)$ \\
\hline & No & 37 & $(24,8)$ & 12 & $(8,1)$ & 18 & $(12,1)$ & 7 & $(4,7)$ \\
\hline \multirow{3}{*}{$\begin{array}{l}\text { Tiempo necesario para } \\
\text { llegar a la IPS }\end{array}$} & & & & & & & & & \\
\hline & $\leq 15$ minutos & 87 & $(58,4)$ & 24 & $(16,1)$ & 44 & $(29,5)$ & 19 & $(12,8)$ \\
\hline & $>15$ minutos & 62 & $(41,6)$ & 20 & $(13,4)$ & 28 & $(18,8)$ & 14 & $(9,4)$ \\
\hline \multirow{3}{*}{$\begin{array}{l}\text { Tiempo entre la petición } \\
\text { de la cita y la fecha de } \\
\text { atención }\end{array}$} & & & & & & & & & \\
\hline & $\begin{array}{l}\leq \text { una } \\
\text { semana }\end{array}$ & 123 & $(82,6)$ & 38 & $(25,5)$ & 58 & $(38,9)$ & 27 & $(18,1)$ \\
\hline & $\begin{array}{l}>\text { una } \\
\text { semana }\end{array}$ & 26 & $(17,4)$ & 6 & $(4,0)$ & 14 & $(9,4)$ & 6 & $(4,0)$ \\
\hline \multirow{2}{*}{$\begin{array}{l}\text { ¿Recibió el tratamiento } \\
\text { para su enfermedad de las }\end{array}$} & & & & & & & & & \\
\hline & $\mathrm{Si}$ & 105 & $(70,5)$ & 34 & $(22,8)$ & 51 & $(34,2)$ & 20 & $(13,4)$ \\
\hline \multirow{2}{*}{$\begin{array}{lcl}\text { encías y } & \text { dientes } \\
\text { (periodontitis)? } & \\
\end{array}$} & No & 42 & $(28,2)$ & 9 & $(6,0)$ & 21 & $(14,1)$ & 12 & $(8,1)$ \\
\hline & No sabe & 2 & $(1,3)$ & 1 & $(0,7)$ & 0 & $(0)$ & 1 & $(0,7)$ \\
\hline \multirow{3}{*}{$\begin{array}{lr}\text { ¿Fue remitido al } & \text { al } \\
\text { especialista } & \text { en } \\
\text { periodoncia? } & \end{array}$} & & & & & & & & & \\
\hline & $\mathrm{Si}$ & 1 & $(0,7)$ & 0 & (0) & 1 & $(0,7)$ & 0 & (0) \\
\hline & No & 148 & $(99,3)$ & 44 & $(29,5)$ & 71 & $(47,7)$ & 33 & $(22,1)$ \\
\hline \multirow{4}{*}{$\begin{array}{l}\text { Después de la valoración } \\
\text { inicial ¿ha podido acceder } \\
\text { al tratamiento } \\
\text { odontológico cuando lo ha } \\
\text { necesitado? }\end{array}$} & & & & & & & & & \\
\hline & $\mathrm{Si}$ & 127 & $(85,2)$ & 39 & $(26,2)$ & 60 & $(40,3)$ & 28 & $(18,8)$ \\
\hline & No & 11 & $(7,4)$ & 4 & $(2,7)$ & 6 & $(4,0)$ & 1 & $(0,7)$ \\
\hline & $\begin{array}{l}\text { No ha } \\
\text { buscado } \\
\text { tratamiento }\end{array}$ & 11 & $(7,4)$ & 1 & $(0,7)$ & 6 & $(4,0)$ & 4 & $(2,7)$ \\
\hline
\end{tabular}


Tabla 4. Distribución porcentual de las variables administrativas de acceso al servicio de odontología según el nivel de severidad de la periodontitis.

\begin{tabular}{|c|c|c|c|c|c|c|c|c|c|}
\hline \multirow{4}{*}{$\begin{array}{l}\text { El tratamiento fue pagado } \\
\text { con }\end{array}$} & & & & & & & & & \\
\hline & $\begin{array}{l}\text { Dinero } \\
\text { propio }\end{array}$ & 3 & $(2,0)$ & 2 & $(1,3)$ & 1 & $(0,7)$ & 0 & (0) \\
\hline & $\begin{array}{l}\text { Préstamo } \\
\text { personal }\end{array}$ & 1 & $(0,7)$ & 0 & $(0,0)$ & 1 & $(0,7)$ & 0 & (0) \\
\hline & EPS & 145 & $(97,4)$ & 42 & $(28,2)$ & 70 & $(47,0)$ & 33 & $(22,2)$ \\
\hline \multirow{5}{*}{$\begin{array}{l}\text { En su opinión el costo del } \\
\text { tratamiento fue }\end{array}$} & & & & & & & & & \\
\hline & Económico & 19 & $(12,8)$ & 4 & $(2,7)$ & 11 & $(7,4)$ & 4 & $(2,7)$ \\
\hline & Justo & 106 & $(71,1)$ & 33 & $(22,1)$ & 49 & $(32,9)$ & 24 & $(16,1)$ \\
\hline & Costoso & 6 & $(4,0)$ & 4 & $(2,7)$ & 2 & $(1,3)$ & 0 & (0) \\
\hline & $\begin{array}{l}\text { No conoce el } \\
\text { costo }\end{array}$ & 18 & (12.1) & 3 & $(2,0)$ & 10 & $(6,7)$ & 5 & $(3,4)$ \\
\hline \multirow{5}{*}{$\begin{array}{l}\text { ¿En cuánto tiempo le } \\
\text { sugirieron regresar a su } \\
\text { tratamiento de dientes y } \\
\text { encías? }\end{array}$} & & & & & & & & & \\
\hline & Una semana & 1 & $(0,7)$ & 0 & (0) & 1 & $(0,7)$ & 0 & $(0)$ \\
\hline & Tres meses & 3 & $(2,0)$ & 0 & (0) & 2 & $(1,3)$ & 1 & $(0,7)$ \\
\hline & Seis meses & 133 & $(89,3)$ & 41 & $(27,5)$ & 65 & $(43,6)$ & 27 & $(18,1)$ \\
\hline & No le dijeron & 12 & $(8,1)$ & 3 & $(2,0)$ & 4 & $(2,7)$ & 5 & $(3,4)$ \\
\hline \multirow{6}{*}{$\begin{array}{l}\text { ¿Qué tan satisfecho está } \\
\text { con el trato recibido por } \\
\text { quien le realizó el } \\
\text { tratamiento? }\end{array}$} & & & & & & & & & \\
\hline & Nada & 0 & $(0)$ & 0 & (0) & 0 & (0) & 0 & (0) \\
\hline & Algo & 3 & $2,4)$ & 1 & $(0,7)$ & 1 & $(0,7)$ & 1 & $(0,7)$ \\
\hline & Indiferente & 2 & $(1,6)$ & 1 & $(0,7)$ & 0 & (0) & 1 & $(0,7)$ \\
\hline & Satisfecho & 104 & $(82,5)$ & 33 & $(26,2)$ & 50 & $(39,7)$ & 21 & $(16,7)$ \\
\hline & $\begin{array}{l}\text { Muy } \\
\text { satisfecho }\end{array}$ & 17 & $(13,5)$ & 4 & $(3,2)$ & 8 & $(6,3)$ & 5 & $(4,0)$ \\
\hline \multirow{6}{*}{$\begin{array}{l}\text { ¿Qué tan satisfecho está } \\
\text { con la forma en que su IPS } \\
\text { atiende sus consultas y } \\
\text { resuelve sus dudas? }\end{array}$} & & & & & & & & & \\
\hline & Nada & 4 & $(2,7)$ & 1 & $(0,7)$ & 2 & $(1,3)$ & 1 & $(0,7)$ \\
\hline & Algo & 8 & $(5,4)$ & 2 & $(1,3)$ & 4 & $(2,7)$ & 2 & $(1,3)$ \\
\hline & Indiferente & 5 & $(3,4)$ & 3 & $(2,0)$ & 1 & $(0,7)$ & 1 & $(0,7)$ \\
\hline & Satisfecho & 115 & $(77,2)$ & 34 & $(22,8)$ & 56 & $(37,6)$ & 25 & $(16,8)$ \\
\hline & $\begin{array}{l}\text { Muy } \\
\text { satisfecho }\end{array}$ & 17 & $(11,4)$ & 4 & $(2,7)$ & 9 & $(6,0)$ & 4 & $(2,7)$ \\
\hline \multirow{6}{*}{$\begin{array}{l}\text { Califique de acuerdo con } \\
\text { todo lo anterior ¿qué tan } \\
\text { satisfecho se siente con la } \\
\text { atención recibida? }\end{array}$} & & & & & & & & & \\
\hline & Nada & 4 & $(2,7)$ & 1 & $(0,7)$ & 2 & $(1,3)$ & 1 & $(0,7)$ \\
\hline & Algo & 7 & $(4,7)$ & 3 & $(2,0)$ & 3 & $(2,0)$ & 1 & $(0,7)$ \\
\hline & Indiferente & 4 & $(2,7)$ & 1 & $(0,7)$ & 2 & $(1,3)$ & 1 & $(0,7)$ \\
\hline & Satisfecho & 121 & $(81,2)$ & 35 & $(23,5)$ & 60 & $(40,3)$ & 26 & $(17,4)$ \\
\hline & $\begin{array}{l}\text { Muy } \\
\text { satisfecho }\end{array}$ & 13 & $(8,7)$ & 4 & $(2,7)$ & 5 & $(3,4)$ & 4 & $(2,7)$ \\
\hline
\end{tabular}


Tabla 5. Distribución porcentual en la segunda medición de la calidad de vida de según los niveles de severidad de la periodontitis.

\begin{tabular}{|c|c|c|c|c|c|c|c|c|}
\hline & \multicolumn{8}{|c|}{ Dimensión Limitación Funcional } \\
\hline & \multicolumn{4}{|c|}{ Problemas pronunciar palabras } & \multicolumn{4}{|c|}{ Peor sabor en la boca } \\
\hline & $\begin{array}{l}\text { Peor } \\
\text { CVRSO }\end{array}$ & $\%$ & $\begin{array}{l}\text { Mejor } \\
\text { CVRSO }\end{array}$ & $\%$ & $\begin{array}{l}\text { Peor } \\
\text { CVRSO }\end{array}$ & $\%$ & $\begin{array}{l}\text { Mejor } \\
\text { CVRSO }\end{array}$ & $\%$ \\
\hline Periodontitis leve & 8 & $(5,4)$ & 36 & $(24,2)$ & 16 & $(10,7)$ & 28 & $(18,8)$ \\
\hline Periodontitis moderada & 8 & $(5,4)$ & 64 & $(43,0)$ & 21 & $(14,1)$ & 51 & $(34,2)$ \\
\hline Periodontitis grave & 10 & $(6,7)$ & 23 & $(15,4)$ & 16 & $(10,7)$ & 17 & $(11,4)$ \\
\hline \multirow[t]{4}{*}{ Total } & 26 & $(17,4)$ & 123 & $(82,6)$ & 53 & $(35,6)$ & 96 & $(64,4)$ \\
\hline & \multicolumn{8}{|c|}{ Dimensión Dolor Físico } \\
\hline & \multicolumn{4}{|c|}{ Molestias dolorosas } & \multicolumn{4}{|c|}{ Inconfortable comer } \\
\hline & $\begin{array}{l}\text { Peor } \\
\text { CVRSO }\end{array}$ & $\%$ & $\begin{array}{l}\text { Mejor } \\
\text { CVRSO }\end{array}$ & $\%$ & $\begin{array}{l}\text { Peor } \\
\text { CVRSO }\end{array}$ & $\%$ & $\begin{array}{l}\text { Mejor } \\
\text { CVRSO }\end{array}$ & $\%$ \\
\hline Periodontitis leve & 31 & $(20,8)$ & 13 & $(8,7)$ & 26 & $(17,4)$ & 18 & $(12,1)$ \\
\hline Periodontitis moderada & 43 & $(28,9)$ & 29 & $(19,5)$ & 39 & $(26,2)$ & 33 & $(22,1)$ \\
\hline Periodontitis grave & 22 & $(14,8)$ & 11 & $(7,4)$ & 21 & $(14,1)$ & 12 & $(8,1)$ \\
\hline \multirow[t]{4}{*}{ Total } & 96 & $(64,4)$ & 53 & $(35,6)$ & 86 & $(57,7)$ & 63 & $(42,3)$ \\
\hline & \multicolumn{8}{|c|}{ Dimensión Malestar Psicológico } \\
\hline & \multicolumn{4}{|c|}{ Consciente de si mismo } & \multicolumn{4}{|c|}{ Sentirse tenso } \\
\hline & $\begin{array}{l}\text { Peor } \\
\text { CVRSO }\end{array}$ & $\%$ & $\begin{array}{l}\text { Mejor } \\
\text { CVRSO }\end{array}$ & $\%$ & $\begin{array}{l}\text { Peor } \\
\text { CVRSO }\end{array}$ & $\%$ & $\begin{array}{l}\text { Mejor } \\
\text { CVRSO }\end{array}$ & $\%$ \\
\hline Periodontitis leve & 42 & $(28,2)$ & 2 & $(1,3)$ & 22 & $(14,8)$ & 22 & $(14,8)$ \\
\hline Periodontitis moderada & 68 & $(45,6)$ & 4 & $(2,7)$ & 26 & $(17,4)$ & 46 & $(30,9)$ \\
\hline Periodontitis grave & 33 & $(22,1)$ & 0 & $(0,0)$ & 14 & $(9,4)$ & 19 & $(12,8)$ \\
\hline \multirow[t]{4}{*}{ Total } & 143 & $(96,0)$ & 6 & $(4,0)$ & 62 & $(41,6)$ & 87 & $(58,4)$ \\
\hline & \multicolumn{8}{|c|}{ Dimensión Incapacidad Física } \\
\hline & \multicolumn{4}{|c|}{ Dieta insatisfactoria } & \multicolumn{4}{|c|}{ Interrumpir comidas } \\
\hline & $\begin{array}{l}\text { Peor } \\
\text { CVRSO }\end{array}$ & $\%$ & $\begin{array}{l}\text { Mejor } \\
\text { CVRSO }\end{array}$ & $\%$ & $\begin{array}{l}\text { Peor } \\
\text { CVRSO }\end{array}$ & $\%$ & $\begin{array}{l}\text { Mejor } \\
\text { CVRSO }\end{array}$ & $\%$ \\
\hline Periodontitis leve & 19 & $(12,8)$ & 25 & $(16,8)$ & 22 & $(14,8)$ & 22 & $(14,8)$ \\
\hline Periodontitis moderada & 23 & $(15,4)$ & 49 & $(32,9)$ & 24 & $(16,1)$ & 48 & $(32,2)$ \\
\hline Periodontitis grave & 10 & $(6,7)$ & 23 & $(15,4)$ & 14 & $(9,4)$ & 19 & $(12,8)$ \\
\hline \multirow[t]{4}{*}{ Total } & 52 & $(34,9)$ & 97 & $(65,1)$ & 60 & $(40,3)$ & 89 & $(59,7)$ \\
\hline & \multicolumn{8}{|c|}{ Dimensión Incapacidad Psicológica } \\
\hline & \multicolumn{4}{|c|}{ Difícil relajarse } & \multicolumn{4}{|c|}{ Sentirse avergonzado } \\
\hline & $\begin{array}{l}\text { Peor } \\
\text { CVRSO }\end{array}$ & $\%$ & $\begin{array}{l}\text { Mejor } \\
\text { CVRSO }\end{array}$ & $\%$ & $\begin{array}{l}\text { Peor } \\
\text { CVRSO }\end{array}$ & $\%$ & $\begin{array}{l}\text { Mejor } \\
\text { CVRSO }\end{array}$ & $\%$ \\
\hline Periodontitis leve & 19 & $(12,8)$ & 25 & $(16,8)$ & 17 & $(11,4)$ & 27 & $(18,1)$ \\
\hline Periodontitis moderada & 23 & $(15,4)$ & 49 & $(32,9)$ & 15 & $(10,1)$ & 57 & $(38,3)$ \\
\hline Periodontitis grave & 14 & $(9,4)$ & 19 & $(12,8)$ & 14 & $(9,4)$ & 19 & $(12,8)$ \\
\hline \multirow[t]{4}{*}{ Total } & 56 & $(37,6)$ & 93 & $(62,4)$ & 46 & $(30,9)$ & 103 & $(69,1)$ \\
\hline & \multicolumn{8}{|c|}{ Dimensión Incapacidad Social } \\
\hline & \multicolumn{4}{|c|}{ Irritable con otras personas } & \multicolumn{4}{|c|}{ Dificultad en su trabajo habitual } \\
\hline & $\begin{array}{l}\text { Peor } \\
\text { CVRSO }\end{array}$ & $\%$ & $\begin{array}{l}\text { Mejor } \\
\text { CVRSO }\end{array}$ & $\%$ & $\begin{array}{l}\text { Peor } \\
\text { CVRSO }\end{array}$ & $\%$ & $\begin{array}{l}\text { Mejor } \\
\text { CVRSO }\end{array}$ & $\%$ \\
\hline Periodontitis leve & 19 & $(12,8)$ & 25 & $(16,8)$ & 10 & $(6,7)$ & 34 & $(22,8)$ \\
\hline Periodontitis moderada & 15 & $(10,1)$ & 57 & $(38,3)$ & 13 & $(8,7)$ & 59 & $(39,6)$ \\
\hline
\end{tabular}


Efecto del acceso al tratamiento de la periodontitis sobre la calidad de vida relacionada con la salud bucal

Continuación de la tabla 5.

\begin{tabular}{|l|l|l|l|l|l|l|l|l|}
\hline Periodontitis grave & 9 & $(6,0)$ & 24 & $(16,1)$ & 8 & $(5,4)$ & 25 & $(16,8)$ \\
\hline Total & 43 & $(28,9)$ & 106 & $(71,1)$ & 31 & $(20,8)$ & 118 & $(79,2)$ \\
\hline \multicolumn{8}{|l|}{} & \multicolumn{2}{l|}{ Incapaz de funcionar } \\
\hline \\
\hline
\end{tabular}

Tabla 6. Distribución final del total de la sumatoria de los rangos de afectación de la CRSB según los niveles de severidad de la periodontitis.

\begin{tabular}{|c|c|c|c|c|c|c|c|c|c|}
\hline & \multicolumn{4}{|c|}{ Método aditivo simple } & \multicolumn{5}{|c|}{ Prueba Kruskal- Wallis } \\
\hline \multirow{4}{*}{$\begin{array}{l}\text { Severidad } \\
\text { de la } \\
\text { periodontitis } \\
\text { según el nivel de } \\
\text { inserción clínica }\end{array}$} & & $\mathbf{N}$ & Media & $\mathrm{DE}$ & $\begin{array}{l}\text { Mediana } \\
\text { global }\end{array}$ & Mín & Máx & \multicolumn{2}{|c|}{$\begin{array}{l}\text { Estadístico } \\
\text { Valor } p\end{array}$} \\
\hline & Leve & 44 & 15,04 & 9,69 & & 0 & 43 & \multirow[t]{4}{*}{5,14} & \multirow[t]{4}{*}{0,076} \\
\hline & Moderada & 72 & 12,00 & 8,11 & & 3 & 43 & & \\
\hline & Grave & 33 & 14,57 & 7,61 & & 4 & 31 & & \\
\hline & Total & 149 & 13,46 & 8,57 & 12 & 0 & 43 & & \\
\hline
\end{tabular}


Tabla 7. Análisis crudo y ajustado variables significativas para peor calidad de vida relacionada con la salud bucal.

\begin{tabular}{|c|c|c|c|c|c|c|c|c|}
\hline & & $\begin{array}{c}\mathrm{RR} \\
\text { (crudo) }\end{array}$ & $\begin{array}{r}\text { Inter } \\
\text { con }\end{array}$ & $\begin{array}{l}\text { los de } \\
\text { anza }\end{array}$ & $\begin{array}{c}R R \\
\text { (ajust) }\end{array}$ & & $\begin{array}{l}\text { los de } \\
\text { anza }\end{array}$ & $\begin{array}{c}\text { Logaritmo } \\
\text { verosimilitud }\end{array}$ \\
\hline & & & Inf & Sup & & $\operatorname{lnf}$ & Sup & \\
\hline Sexo & & & & & & & & \\
\hline & Masculino & 1 & & & & & & 195,630 \\
\hline & Femenino & 2,15 & 1,14 & 4,06 & 1,962 & 1,020 & 3,775 & \\
\hline Ocupación & & & & & & & & \\
\hline & Empleado & 1 & & & & & & 195,792 \\
\hline & Desempleado & 1,61 & 0,519 & 5,01 & 1,724 & 0,548 & 2,425 & \\
\hline & Hogar & 2,54 & 0,897 & 7,20 & 2,187 & 0,785 & 6,097 & \\
\hline & Independiente & 2,21 & 0,749 & 6,50 & 2,909 & 0,977 & 8,660 & \\
\hline & Estudiante & 3,13 & 0,994 & 9,83 & 2,516 & 0,744 & 8,509 & \\
\hline Severidad & & & & & & & & \\
\hline periodontitis & Leve & 1 & & & & & & 194,525 \\
\hline & Moderada & 0,538 & 0,349 & 0,829 & 0,626 & 0,408 & 0,961 & \\
\hline & Grave & 0,907 & 0,596 & 1,379 & 0,814 & 0,520 & 1,274 & \\
\hline Uso de prótesis & & & & & & & & \\
\hline dental & No & 1 & & & & & & 194,722 \\
\hline & $\mathrm{Si}$ & 2,22 & 1,630 & 3,02 & 1,571 & 1,024 & 2,411 & \\
\hline Pérdidas & & & & & & & & \\
\hline dentales & No & 1 & & & & & & 203,150 \\
\hline & $\mathrm{Si}$ & 1,16 & 0,740 & 1,81 & 0,823 & 0,487 & 1,392 & \\
\hline Movilidad & & & & & & & & \\
\hline dental, lesión & No & 1 & & & & & & 203,228 \\
\hline $\begin{array}{l}\text { de furca, o } \\
\text { supuración }\end{array}$ & $\mathrm{Si}$ & 1,19 & 0,700 & 2,02 & 1,068 & 0,571 & 1.997 & \\
\hline Sentirse tan mal & & & & & & & & \\
\hline con su & No & 1 & & & & & & 195,856 \\
\hline $\begin{array}{l}\text { condición oral } \\
\text { que ha llorado }\end{array}$ & $\mathrm{Si}$ & 1,71 & 1,15 & 2,55 & 1,296 & 0,863 & 1,948 & \\
\hline Acomplejado & & & & & & & & \\
\hline por sus dientes & No & 1 & & & & & & 185,630 \\
\hline y/o encías & $\mathrm{Si}$ & 0,458 & 0,327 & 0,641 & 0,600 & 0,371 & 0,972 & \\
\hline Considerar que & & & & & & & & \\
\hline su boca tiene & $\mathrm{Si}$ & 1 & & & & & & 197,961 \\
\hline $\begin{array}{l}\text { buena } \\
\text { apariencia }\end{array}$ & No & 1,56 & 1,09 & 2,24 & 0,977 & 0,603 & 1,585 & \\
\hline Sentirse & & & & & & & & \\
\hline satisfecho & $\mathrm{Si}$ & 1 & & & & & & 196,293 \\
\hline consigo mismo & No & 2,16 & 1,56 & 3,01 & 1,561 & 0,890 & 2,738 & \\
\hline Recibir & & & & & & & & \\
\hline tratamiento & $\mathrm{Si}$ & 1 & & & & & & 199,767 \\
\hline para la & No & 0,870 & 0,559 & 1,35 & 0,837 & 0,559 & 1,252 & \\
\hline periodontitis & No sabe & 2,283 & 1,838 & 2,83 & 1,683 & 0,789 & 3,591 & \\
\hline De acuerdo con & & & & & & & & 202,674 \\
\hline todo lo anterior & Muy satisfecho & 1 & & & & & & \\
\hline ¿qué $\quad \tan$ & Satisfecho & 0,895 & 0,480 & 1,67 & 0,932 & 0,502 & 1,731 & \\
\hline satisfecho se & Indiferente & 1,083 & 0,346 & 3,40 & 1,088 & 0,354 & 3,344 & \\
\hline siente con la & Algo & 1,238 & 0,519 & 2,95 & 0,876 & 0,381 & 2,013 & \\
\hline $\begin{array}{l}\text { atención } \\
\text { recibida? }\end{array}$ & Nada & 1,083 & 0,346 & 3,40 & 1,776 & 0,524 & 6,021 & \\
\hline
\end{tabular}




\section{DISCUSIÓN}

En este estudio, se utilizó como referente el modelo de acceso a los servicios de salud planteado por Andersen ${ }^{5}$, probado en relación con el cuidado de la salud bucal como lo describen primero Baker et a ${ }^{19}$ al examinaron las vías directas e indirectas entre los factores sociales, de actitud y comportamentales asociados a la percepción de la salud bucal; luego Marshman et $a l^{20}$, en un estudio que buscaba identificar los factores que influencian la utilización de los servicios odontológicos; aunque los resultados estaban alineados con el modelo, sus conclusiones indicaron la necesidad de incluir otros factores para aumentar su utilidad en la comprensión del acceso a los servicios odontológicos y los resultados de la salud bucal. Esto fue confirmado en el estudio de Holde et $a l^{21}$, donde incluyeron variables complementarias.

En este estudio, los participantes respondieron que el rango de tiempo entre la solicitud de la atención y el momento de la misma era menor a una semana, esto puede estar relacionado con el elevado porcentaje de pacientes que refirieron haber recibido el tratamiento de la enfermedad periodontal (70,5\%), además del subsidio del servicio de odontología general; lo cual permite que su percepción sea que el costo del tratamiento sea justo o económico en la mayoría de los casos, y que solo el $4 \%$ lo considere costoso cuando debe pagar una cuota moderadora de $\$ 3.400$ (USD $\$ 0,906$ / tasa de cambio del día 12 de mayo del 2021 donde 1 USD=3.750 COP).

Aunque se informó a los pacientes la necesidad de recibir tratamiento periodontal especializado, al finalizar el tiempo de seguimiento y encontrar que los participantes no buscaban acceder al manejo sugerido, se decidió preguntarles las razones por las cuales no intentaron acceder al especialista como variable emergente ante la curiosidad de los investigadores, la mayoría de las respuestas estuvieron dirigidas hacia las limitaciones financieras, el no tener tiempo disponible, pero algunos respondían que no lo consideraban necesario por no sentir dolor, o no les interesaba. Estos resultados difieren de los hallados por Lima
Carreiro et $a l^{22}$, quienes utilizaron bases de datos municipales públicas al medir la insatisfacción con los servicios odontológicos de acuerdo con los parámetros establecidos por el modelo teórico de Andersen, encontraron que el $14,9 \%$ estaba insatisfecho con el servicio, el grupo con mayor proporción de insatisfacción estaba compuesto por personas con mayor nivel educativo y procedentes de municipios con mayor índice de inequidades sociales.

Un estudio con resultados similares a los de esta investigación, fue realizado por Singh A. et $a l^{23}$, en la población de New Dheli (India), quienes estaban cubiertos por el seguro odontológico del Plan Nacional de Seguridad Social de la India; allí la mayoría de los participantes estuvieron satisfechos con la atención recibida en la recepción del hospital $(74,7 \%)$; sin embargo, reportaron retrasos mayores a un mes en las citas agendadas $(37,9 \%)$, espera superior a una hora para ser atendidos $(88,6 \%)$, la mayoría reportó estar insatisfecho con la atención recibida por parte de la ayudante del odontólogo tratante en un $76,7 \%$, con el odontólogo el $51 \%$, pero en general la satisfacción con la experiencia del servicio de odontología fue del $64,5 \%$. Otros estudios desarrollados en instituciones privadas de atención especializada, evidenciaron que los pacientes con mayor nivel educativo y edades superiores al promedio, reportan menos satisfacción con los servicios odontológicos ${ }^{24,25}$.

En este estudio, nueve participantes usaban prótesis dental (6\%) y a pesar de todos tener diagnóstico de periodontitis, aunque muy pocos con el diagnóstico en su historia clínica, la mayoría de los participantes $(37,6 \%)$ afirmaron tener más de un año desde su última visita al odontólogo, mientras el $18,1 \%$ refirió haber asistido hace un año, el $12,8 \%$ asistió por última vez un mes antes del ingreso al estudio y finalmente el 10,7\% asistieron seis meses antes. La baja frecuencia de visitas al servicio de odontología puede deberse a las necesidades específicas de tratamiento, dado que al no contar con el registro del diagnóstico de periodontitis tanto operador como paciente desconocen la necesidad de tratamiento, como lo describen Valdez et $a l^{26}$, encontraron asociación entre las visitas frecuentes 
al odontólogo y el menor nivel de bienestar, porque los usuarios asiduos requerían en su mayoría procedimientos restauradores, endodónticos y protésicos, pero al mismo tiempo eran los usuarios con mayor porcentaje de caries y bolsas periodontales al compararlos con los menos asiduos; las visitas frecuentes al servicio de odontología se asociaron con una menor satisfacción con la vida $\mathrm{OR}=0,89(\mathrm{IC}=0,80 ; 0,99)$ y con sentimientos negativos como la tristeza y la ansiedad $\mathrm{OR}=1,41$ (IC=1,22;1,64). Los hallazgos de Valdez $\mathrm{R}$ et al concuerdan con los descritos por Nihtila A. et $\left.a\right|^{27,28}$ al encontrar que el $61,6 \%$ de los usuarios tenían alta frecuencia de asistencia al servicio de odontología.

En esta investigación, diferencia de los estudios de Nihtila et al, los participantes no acudían con frecuencia al odontólogo; las variables asociadas a la peor CVRSB fueron sexo y uso de prótesis dental (ver tabla 7), además el procedimiento realizado con mayor frecuencia fue la higiene oral $(76,5 \%)$. Se encontraron caries activas en el $32,9 \%$ y pérdidas dentales en el $73,8 \%$ sin incluir cordales; la baja complejidad de los procedimientos odontológicos ofrecidos en la IPS donde se realizó la investigación por ausencia de especialidades coincide con lo expuesto por Northridge $M$. et $a l^{29}$ quienes consideran imperativo eliminar la disparidad en la atención de la salud bucal. Es necesario investigar las inequidades de forma profunda en todos los aspectos de la atención en salud bucal, incluir la asignación de recursos, recepción real de los servicios, calidad de los procesos ${ }^{30}$ y financiación con respecto a la carga de pago de las personas y los hogares $^{31}$, por ser las personas con menores recursos quienes hasta el momento no han recibido suficiente atención por parte de la salud pública.

En este estudio se encontró que los participantes en general presentaron una mejor CVRSB a pesar del nivel de severidad de la periodontitis; en la primera medición el 53\% presentó una peor calidad de vida, mientras en la segunda medición el 42,9\% tuvo una peor CVRSB, el grupo con periodontitis grave refirió con mayor frecuencia una peor calidad de vida; estos hallazgos son similares con los reportados por Al Habashneh et $a^{32}$ al encontrar peor CVRSB en pacientes con periodontitis moderada (54\%) y periodontitis grave $(64 \%)^{33}$. Wong et al ${ }^{34}$ en su umbrella review describe la enfermedad periodontal como un factor negativo sobre la calidad de vida, pero también incluye autores que reportan resultados. Hasta el momento, no se ha encontrado en la literatura de reportes de pacientes con periodontitis que no hayan logrado acceder al tratamiento de la enfermedad por personal entrenado y con los equipos e instrumental destinados para esa finalidad, especialmente al medir la CVRSO, lo que permite considerar este estudio posiblemente el primero en esta área.

Recibir tratamiento no especializado para el tratamiento de la enfermedad periodontal en el servicio de odontología del centro de salud, hace que el paciente perciba que accede al tratamiento y no perciba las barreras administrativas $y$ organizacionales. La población participante en esta investigación no refiere sentirse acomplejada por su condición bucal y en general está satisfecha consigo misma; por lo cual, su distribución de mejor y peor CVRSB son similares. No contar con atención especializada en el centro de salud puede influir en sus expectativas acerca del tratamiento; esto puede explicarse desde la representación social de la salud bucal como un estado de salud adaptado de la teoría ecológica de los sistemas de Urien Bronfenbrenner ${ }^{34}$, que explica cómo el entorno de crecimiento es determinante y afecta la forma de pensar, emociones y gustos; al aplicarlo al área de la odontología y al tratamiento de la periodontitis deben considerarse las actitudes relacionadas con lo cognitivo, porque al no conocer la enfermedad no tienen preocupación; también actitud desde lo afectivo, donde la persona puede considerar que las pérdidas dentales, movilidad u otros síntomas de la periodontitis pueden considerarse normales por la edad cumplida. Lo anterior, converge en la actitud conductual, donde al tener el desconocimiento de la enfermedad y considerarla parte del proceso fisiológico del envejecimiento no es imperativo buscar la atención preventiva o especializada para el tratamiento de la periodontitis, porque no influye en el reconocimiento de cómo es percibida la persona en su comunidad cuando presenta las pérdidas dentales u otras secuelas.

Los cambios actitudinales y comportamentales en lo referente al proceso salud-enfermedad bucal desde la epidemiología social requiere considerar la 
construcción de modos de vida, colectivos que produzcan bienestar dentro de las comunidades; repensar y cuestionar la práctica odontológica al pensar en el desarrollo de saberes colectivos que permitan influir e intentar modificar pautas culturales preexistentes en el personal de la salud y en la comunidad en general. Por eso, la salud bucal colectiva, no debe desvincularse del escenario político integrando saberes desde un análisis sociocultural e histórico de cada población de manera que se incluyan los distintos actores sociales y sus interacciones ${ }^{36}$.

\section{CONCLUSIÓN}

En pacientes que asisten a tratamiento odontológico en un centro de salud del régimen subsidiado, donde predomina la población con nivel bajo nivel educativo y con predominio de mujeres dedicadas a las labores del hogar, no se encontró asociación causal entre el acceso al tratamiento de la periodontitis y el efecto sobre la peor CVRSB, por estar los participantes satisfechos con el servicio recibido. La mayoría de los participantes no presentaron sintomatología asociada a la periodontitis y perciben la movilidad y pérdida dental como parte de su proceso de envejecimiento; además de no considerar que afecta su desempeño social.

La primera barrera para acceder al tratamiento de la periodontitis es la ausencia de diagnóstico de la enfermedad por parte del personal del servicio de odontología, de los pacientes y de los tomadores de decisiones, lo que hace esta barrera imperceptible. Otra barrera emergente durante la observación de los investigadores fue la corrupción, y su efecto sobre la oferta de los servicios de salud antes de ser intervenidos por la Superintendencia Nacional de Salud.

\section{DECLARACIÓN SOBRE CONFLICTOS DE INTERÉS}

Este proyecto fue financiado por la Dirección de Investigación e Innovación de la Universidad CES en Medellín y por la Universidad del Magdalena en Santa Marta; los autores declaran que en la redacción del manuscrito no incidieron intereses o valores distintos a los que usualmente tiene la investigación.

\section{CONTRIBUCIÓN DE LOS AUTORES}

Primer autor: diseño del estudio, gestión financiación, trabajo de campo, escritura, revisión y aprobación.

Segundo autor: escritura, revisión y aprobación Tercer autor: escritura, revisión y aprobación.

\section{REFERENCIAS BIBLIOGRÁFICAS}

1. Gift HC, Atchison KA, Dayton CM. Conceptualizing oral health and oral health-related quality of life. Soc Sci Med. 1997;44(5):601-8. DOI: 10.1016/s02779536(96)00211-0

2. O'Dowd LK, Durham J, McCracken GI, Preshaw PM. Patients' experiences of the impact of periodontal disease. J Clin Periodontol. 2010;37(4):334-9. DOI: 10.1111/j.1600051X.2010.01545.x

3. Kassebaum NJ, Bernabé E, Dahiya M, Bhandari B, Murray CJL, Marcenes W. Global burden of severe periodontitis in 1990-2010: a systematic review and meta-regression. J Dent Res. 2014;93(11):1045-53. DOI: $10.1177 / 0022034514552491$

4. República de Colombia. Ministerio de salud y protección social. IV Estudio nacional de salud bucal ENSAB IV 2013-2014. Disponible en: https://www.minsalud.gov.co/sites/rid/Lists/Bibliot ecaDigital/RIDE/VS/PP/ENSAB-IV-Situacion-Bucal-

Actual.pdf

5. Andersen R. AL. A framework for the study of acces to medical care. Health services research. 1974;208-20. Disponible en: https://www.ncbi.nlm.nih.gov/pmc/articles/PMC10 71804/

6. Glazier R., Sibley L. Reasons for self-reported unmet healthcare needs in Canada: a populationbased provincial comparison. Healthcare policy. 2009;5(1):87-104. Disponible en: https://www.ncbi.nlm.nih.gov/pmc/articles/PMC27 32657/ 
7. Masseria C., Allin S. Unmet needs as an indicator of health care access. Eurohealth. 2009;15(3):7-9. Disponible en: https://www.cabdirect.org/cabdirect/abstract/201 03049399

8. González L. Orientaciones de lectura sobre vulnerabilidad social. Disponible en: http://biblioteca.clacso.edu.ar/Argentina/ceaunc/20100825121333/Lecturasvulnerabilidad.pdf

9. Moreno Crossley JC. El concepto de vulnerabilidad social en el debate en torno a la desigualdad: problemas, alcances y perspectivas. Disponible en: http://www.sitemason.com/files/h2QrBK/WORKIN G\%20PAPERS\%209.pdf

10. Lopez R, Baelum V. Spanish version of the Oral Health Impact Profile (OHIP-Sp). BMC Oral Health. 2006;6:11. DOI: 10.1186/1472-6831-6-11

11. Loaiza Echeverri ET, Arroyave Zuluaga ID, De La Hoz Perafán R. Evaluación de las propiedades psicométricas de reproducibilidad del Oral Health Impact Profile (ohip-49) entre pacientes con enfermedad periodontal. Rev nac odontol. 2019;15(29):1-27.

DOI: https://doi.org/10.16925/2357-

4607.2019.02.12

12. De La Hoz RP, Segura-Cardona A. Instrumentos para medir la calidad de vida relacionada con la salud oral: una revisión sistemática. Salud Uninorte. 2017;33(3):504-16. DOI:

http://dx.doi.org/10.14482/sun.33.3.10919

13. Slade GD. Derivation and validation of a shortform oral health impact profile. Community Dent Oral Epidemiol. 1997;25(4):284-90. DOI: 10.1111/j.1600-0528.1997.tb00941.x.

14. Montero J, Lopez Jf, Vicente Mp, Galindo Mp, Albaladejo A, Bravo M. Comparative validity of the OIDP and OHIP-14 in describing the impact of oral health on quality of life in a cross-sectional study performed in Spanish adults. Med Oral. 2011;e81621. DOI: $10.4317 /$ medoral.16851
15. International Test Commission. The ITC Guidelines for Translating and Adapting Tests (Second edition). Disponible en: https://www.intestcom.org/files/guideline_test_ad aptation_2ed.pdf

16. Eke PI, Page RC, Wei L, Thornton-Evans G, Genco RJ. Update of the case definitions for populationbased surveillance of periodontitis. J Periodontol. 2012;83(12):1449-54.

DOI: 10.1902/jop.2012.110664

17. Allen FP, Locker D. Do item weights matter? An assessment using the oral health impact profile. Community Dent Health. 1997;14:133-8. Disponible en: https://pubmed.ncbi.nlm.nih.gov/9332036/

18. Robinson PG, Gibson B, Khan FA, Birnbaum W. Validity of two oral health-related quality of life measures. Commun Dent Oral Epidemiol. 2003;31(2):90-9. DOI: $10.1034 /$ j.16000528.2003.00051.x

19. Baker SR. Applying Andersen's behavioural model to oral health: what are the contextual factors shaping perceived oral health outcomes?: Applying Andersen's behavioural model to oral health. Community Dentistry and Oral Epidemiology. 2009;37(6):485-94. DOI: 10.1111/j.1600-0528.2009.00495.x

20. Marshman Z, Porritt J, Dyer T, Wyborn C, Godson J, Baker S. What influences the use of dental services by adults in the UK? Community Dent Oral Epidemiol. 2012;40(4):306-14. DOI: 10.1111/j.1600-0528.2012.00675.x

21. Holde GE, Baker SR, Jönsson B. Periodontitis and quality of life: What is the role of socioeconomic status, sense of coherence, dental service use and oral health practices? An exploratory theory-guided analysis on a Norwegian population. J Clin Periodontol. julio de 2018;45(7):768-79. DOI: $10.1111 /$ jcpe.12906

22. Carreiro DL, Oliveira RFR, Coutinho WLM, Martins AME de BL, Haikal DS. Avaliação da satisfação com a assistência odontológica na perspectiva de usuários brasileiros adultos: análise multinível. Ciênc saúde coletiva. 2018;23(12):4339- 
$49 . \quad$ DOI: https://doi.org/10.1590/1413812320182312.32792016

23. Singh A, Purohit BM. Exploring patient satisfaction levels, self-rated oral health status and associated variables among citizens covered for dental insurance through a National Social Security Scheme in India. International Dental Journal. 2017;67(3):172-9. DOI: 10.1111/idj.12285

24. Rocha J, Pinto A, Batista M, Paula JS de, Ambrosano $\mathrm{G}$. The importance of the evaluation of expectations and perceptions to improve the dental service quality. IJHCQA. 2017;30(6):568-76. DOI: 10.1108/IJHCQA-01-2016-0008

25. Lahti S, Tuutti H, Hausen H, Kaarianen R. Patients' expectations of an ideal dentist and their views concerning the dentist they visited: do the views conform to the expectations and what determines how well they conform? Commun Dent Oral Epidemiol. 1996;24(4):240-4. DOI: 10.1111/j.1600-0528.1996.tb00852.x.

26. Valdez R, Aarabi G, Spinler K, Walther C, Seedorf $U$, Heydecke G, et al. Association between Subjective Well-Being and Frequent Dental Visits in the German Ageing Survey. IJERPH. 2020;17(9):3207. DOI: 10.3390/ijerph17093207

27. Nihtila A., Widstrom E., Elonheimo O. Heavy consumption of dental services among Finnish adults. Community Dent Health. 2010;27:227-32. Disponible en: https://pubmed.ncbi.nlm.nih.gov/21473358/

28. Nihtilä A, Widström E, Elonheimo O. Heavy consumption of dental services; a longitudinal cohort study among Finnish adults. BMC Oral Health. 2013;13(1):18. DOI: 10.1186/1472-6831-1318

29. Northridge ME, Kumar A, Kaur R. Disparities in Access to Oral Health Care. Annu Rev Public Health. 2020;41(1):513-35. DOI: 10.1146/annurevpublhealth-040119-094318

30. Correa MB, Peres MA, Peres KG, Horta BL, Barros AJ, Demarco FF. Do socioeconomic determinants affect the quality of posterior dental restorations? A multilevel approach. Journal of Dentistry. 2013;41(11):960-7.

DOI: 10.1016/j.jdent.2013.02.010

31. Vujicic M, Buchmueller T, Klein R. Dental Care Presents The Highest Level Of Financial Barriers, Compared To Other Types Of Health Care Services. Health Affairs. 2016;35(12):2176-82. DOI: $10.1377 /$ hlthaff.2016.0800

32. Al Habashneh R, Khader YS, Salameh S. Use of the Arabic version of Oral Health Impact Profile-14 to evaluate the impact of periodontal disease on oral health-related quality of life among Jordanian adults. J Oral Sci. 2012;54(1):113-20. DOI: 10.2334/josnusd.54.113

33. Costa FO, Guimar\&atilde; es AN, Cota LOM, Pataro AL, Segundo TK, Cortelli SC, et al. Impact of different periodontitis case definitions on periodontal research. Journal of Oral Science. 2009;51(2):199-206. DOI: 10.2334/josnusd.51.199

34. Wong LB, Yap AU, Allen PF. Periodontal disease and quality of life: Umbrella review of systematic reviews. J Periodont Res. 2021;56(1):1-17. DOI: 10.1111/jre.12805

35. Bronfenbrenner U. La ecología del desarrollo humano. 1ra ed. Barcelona: Ediciones Paidós Ibérica; 1987. 31 p. Disponible en: http://proyectos.javerianacali.edu.co/cursos_virtua les/posgrado/maestria_asesoria_familiar/familia_c ontemporanea/modulo1/la-ecologia-deldesarrollo-humano-bronfenbrenner-copia.pdf

36. Beltrán-Salazar M. De lo oral a lo bucal en la odontología. Revista Colombiana de Investigación en Odontología. 2011;2(5):1-7. Disponible en: https://acfo.edu.co/ojs/index.php/rcio/rt/printerFr iendly $/ 58 / 0$ 